\title{
Management of Musculoskeletal Pain
}

\author{
Dina Soliman ${ }^{1,2}$ \\ 1. Rheumatology and Rehabilitation, Ain Shams University, Cairo 11566, Egypt \\ 2. Board certified in Regenerative Medicine, American Academy of Regenerative Medicine, Denver 80401, USA
}

\begin{abstract}
Musculoskeletal pain is a distressing, unpleasant feeling and an emotional experience associated with actual or potential tissue damage. Prolotherapy is an injection therapy designed to stimulate tissue healing. Lyftogt PIT (perineural injection therapy) treats the neurogenic inflammation. Therefore it stops the pain and promotes the release of NGFs (nerve growth factors) which are important for tissue regeneration. Vitamin D3 receptors are ubiquitous in tissue distribution, which opens the possibility for unforeseen biological functions of vitamin D endocrine system. Dextrose is the most commonly used tissue proliferant in prolotherapy. It has different ways of action aiming to stimulate the tissue regeneration. Platelet Rich Plasma is also used in the IROM (interventional regenerative orthopedic medicine), and it is very powerful because it delivers "growth-factors" which stimulate the local tissue progenitor cells (adult mesenchymal stem cells) enhancing the regeneration of the tissues. Adult Mesenchymal stem cell is the hot topic nowadays in the non-surgical field of pain management. In this paper, 250 patients suffering from chronic pain and dysfunction of different body joints (knee, hip and elbow), were treated with one or two injection therapy techniques, to evaluate the patient improvement regarding the pain and function. The effect of adding an intra-articular injection of vitamin D to the above techniques was also evaluated regarding the joint pain and function improvement. Some of the patients were treated with Platelet Rich Plasma and others were treated with Bone Marrow Stem cells. The results of the 250 patients were compared with those obtained from 30 matching control patients, having same conditions and were treated with physiotherapy. Conclusions show that the therapeutic benefit from combining the two techniques, Lyftogt Perineural Injection Therapy and Prolotherapy, is better than using only one of them regarding the pain and function for the different body joints. Adding an intra-articular injection of vitamin D to the treatment of some patients gave a better result than those treated with dextrose only. However, using the Platelet Rich Plasma and the Stem cells instead of dextrose gave the best results ever regarding the pain and function of the musculoskeletal system.
\end{abstract}

Key words: Dextrose prolotherapy, Lyftogt PIT (perineural injection therapy), vitamin D, Platelet Rich Plasma, stem cells.

\section{Introduction}

A wide range of potential multidisciplinary therapies has been advocated for treatment of musculoskeletal pain with an enormous cost to the health care system and to the community but they are not necessarily with strong supportive evidence, and a recent systematic review reported no clear benefit of any one therapy.

Over the years, the pain management has been consisted in the Physical Therapy, while different studies reported that the physical modalities have shown either a short term pain relief or ineffectiveness in treating OA, or no significant difference between patients treated with physiotherapy and placebo group $[1,2]$.

Corresponding author: Dina Soliman, M.Sc., research field: non-surgical interventional regenerative orthopedic medicine.
Non-surgical IROM (interventional regenerative orthopedic medicine) (Prolotherapy and Lyftogt Perineural Injection Therapy) is a method of injection treatment for musculoskeletal pain management.

Prolotherapy is a fundamental and an effective injection therapy for the repair of the injured fibrous connective tissue [3] that holds our skeletal infrastructure together, including the ligaments, tendons, muscle fascia, joint capsular tissue and cartilage on the inside of joints. It is based on the premise that chronic musculoskeletal pain is due to the inadequate repair of the fibrous connective tissue (connective tissue insufficiency) resulting in ligament and tendon weakness or relaxation (laxity) [4].

Prolotherapy has been used in a form recognizable to contemporary practitioners for at least 75 years. Literature from 1930's to 2000's reported positive 
clinical outcomes and that it is the treatment of choice for musculoskeletal injuries and OA especially if the patient fails to improve after physical therapy, chiropractice or orthopathic manipulation, steroid injections, radiofrequency denervation or surgical interventions or if such modalities are contraindicated [5].

Hippocrates, father of modern medicine, said that healing is a matter of time but sometimes is a matter of opportunity, and George S. Hackett, father of prolotherapy, said that prolotherapy acts by stimulating the production of new fibrous tissue and bone cells that will strengthen the "weld" at the fibro-osseous junction. He was the one who developed the prolotherapy technique, formalized its protocol and he was pioneer in Ref. [6].

Lyftogt PIT (perineural injection therapy) is a superficial injection treatment around the cutaneous inflamed nerves and at the sites of their constriction where these nerves become vulnerable to neurogenic inflammation. Hilton's law 1879 stated that treating the superficial nerves eventually treats the underlying joint [7].

The most important molecule in initiating and maintaining pain experience in humans is the TRPV1 (transient receptor potential vanilloid type 1). These molecules are located on the nervi-nervorum of the peripheral nervous system. They are non-specific membrane cation channels allowing the influx of $\mathrm{Na}^{+}$ and $\mathrm{Ca}^{2+} \cdot \mathrm{Na}^{+}$influx results in spike formation and increased action potential causing the neuropathic pain, and $\mathrm{Ca}^{+}$influx triggers the release of the neuropeptides such as substance $\mathrm{P}$ and calcitonin gene related peptide causing the neurogenic inflammation [4].

Dextrose is the most popular solution used in IROM, it is a tissue proliferant having different ways of action aiming to stimulate the tissue regeneration. It acts by: (a) Osmotic shock (glucose 15\%) dehydrating the cells and releasing the intracellular fragments to the extracellular fluid attracting the granulocytes, as well as putting the arachidonic acid pathway on fire [8]; (b) a desirable nutrient gradient which attracts mobile cell [8]; (c) blocks the TRPV1 molecules (glucose 5\%) and leads to a rapid reduction of pain within 10-20 seconds which ends up by a down-regulation of these molecules [9], in addition glucose stimulates the normal flow of nerve growth factors and subsequently allows tissue and nerve repair [10], (d) genes for growth factor production are activated within 20 minutes after exposure of the human cells to glucose [11].

Vitamin D is not strictly a vitamin. It may be considered a hormone as its synthesis and activity occur in different locations [12]. Vitamin D has an unforeseen biological endocrine system functions as its receptors are ubiquitous in tissue distribution. It regulates an extensive number of RNA polymerase II-transcribed genes including genes that govern growth factors release for normal tissue growth and regeneration, oncogene, lymphokine expression, genes involved in mineral homeostasis, vitamin D metabolism, and regulation of a set of replication-linked genes which are critical for rapid cellular proliferation (DNA replication-differentiation switch) [13].

Researchers found that vitamin D and IGF-1 (insulin-like growth factor-1) are involved in a complex interplay that may have implications for growth hormone dosing. In a review of the literature, patients with growth hormone deficiency who had higher vitamin D levels were more likely to achieve serum IGF-1 levels closer to the normal range, and these patients were also more likely to be treated with lower doses of growth hormone. Vitamin $\mathrm{D}$ has been shown to increase circulating IGF-1 and IGF binding proteins (IGFBP-3), while administration of growth hormone and IGF-1 has been shown to increase vitamin D levels in several studies [14].

PRP (platelet rich plasma) is also used in IROM and is very powerful because it delivers "growth-factors" which stimulate the local tissue 
progenitor cells (mesenchymal stem cells) enhancing the regeneration of the tissues. Research studies and clinical practice have shown PRP therapy to be very effective at relieving pain and returning patients to their normal lives. Both ultrasound and MRI images have shown definitive tissue repair after PRP therapy, confirming the healing process.

Stem cells are the hot topic nowadays in the non-surgical field of pain management as they have showed great results in the treatment of musculoskeletal pain and degenerative diseases. They are derived either from bone marrow or from the adipose tissue, and they serve as a sort of internal repair system having the remarkable potential to divide in order to replenish other different cell types.

\section{Analysis}

Over the last three years, 250 patients have been treated for their musculoskeletal pain and OA with Non-surgical IROM (interventional regenerative orthopedic medicine). They were divided to the following groups:

Group 1: (a) 100 patients were treated with Dextrose Prolotherapy.

(b) Lyftogt Perineural Injection Therapy was added to 50 patients of them.

Group 2: Intra-articular injection of vitamin D was added to 25 patients of group (1a) and 25 patients of group (1b).

Group 3: 50 patients were treated with Platelet Rich Plasma.

Group 4: 50 patients were treated with Bone Marrow Stem cells.

The assessment was done using Visual Analogue Scale (pain) and Western Ontario and McMaster Universities osteoarthritis index (function) [15]. This was done for every patient before the start of the treatment, and on each time the patient came to get the treatment session (interval between the sessions depends on the type of treatment used for the patient).

The results of the 250 patients in the above mentioned groups were compared with the results of 30 patients treated with Physiotherapy only. Also the history of 50 patients who went through surgical procedures was reviewed in the scoop of the present results.

\section{Conclusions}

Patients with musculoskeletal pain and OA of different joints in the body treated with physiotherapy did not get the satisfying improvement regarding the decrease in VAS and the increase in function.

Non-surgical IROM (interventional regenerative orthopedic medicine) is the bridge to the future. It helps in regenerating and reconstructing the tissues and the joints without changing neither the stress distribution of the line of pressure nor the biotensegrity of the body. Biotensegrity is defined by the dependence of the different body parts on each other. These parts work simultaneously with each other (compression-tension), which makes the different body parts handle different movement.

The deep technique of IROM-Prolotherapy treatment-for musculoskeletal pain and joint OA is a conservative treatment that gives positive results for the patients. It improves tissue healing, and hence improves the articular cartilage, ligament and tendon repair. Consequently, it decreases the pain (VAS) and increases the function (WOMAC).

The superficial technique of IROM-Lyftogt Perineural Injection Treatment-is the challenging ease that alleviates the pain of the patient immediately by blocking the TRPV1 molecules, and hence decreases the neurogenic inflammation. Therefore, the neuropathic pain and the muscle spasm decrease, and the musculoskeletal function increases.

Patients treated with Prolotherapy and Lyftogt Perineural Injection Therapy techniques in group (1b), showed quicker and better improvement than those in group (1a) treated with Prolotherapy technique only.

Patients in group (2) treated with intra-articular injection of vitamin $\mathrm{D}$ showed a better improvement 
than those treated without it.

Adding an intra-articular injection of vitamin $\mathrm{D}$ to patients suffering from musculoskeletal pain and joint $\mathrm{OA}$, is an easy and simple technique recommended to be used in the poor, rural areas where there are no high technologies making people capable of using other powerful treatments.

Using Platelet Rich Plasma in group (3) and Bone Marrow Adult Stem Cells in group (4) for treatment of musculoskeletal pain and joints gives the patients the quicker and best results regarding the pain and function. However, the use of the adult mesenchymal stem cells in this technique gives the best results even with the most severe and late cases.

Patients who underwent surgeries, most probably, had to go through the surgical procedures again, either in the same region or in another area due to the creation of new extra-stresses in the body after the surgery. These new additional stresses are resulted from the changes in the internal line of body pressure after the surgical procedure. This is due to the changes in the center of gravity of the body parts subjected to surgery, and subsequently other areas will be vulnerable to injuries. Accordingly, the body adapts a new abnormal biomechanical pattern to accommodate the new body situation in order to enable its movement, which puts the musculoskeletal system in a vicious circle of pain re-generation, and the patient never goes back to normal.

\section{References}

[1] Ainsworth, R. 2007. “A Prospective Double Blind Placebo-Controlled Randomized Trial of Ultrasound in the Physiotherapy Treatment of Shoulder Pain." Rheumatology 46 (5): 815-20.

[2] Brosseau, L., Casimiro, L., Robinson, V., Milne, S., Shea, B., Judd, M., Wells, G., and Tugwell, P. 2001.
"Therapeutic Ultrasound for Treating Patello-Femoral Pain Syndrome.” Cochrane Database Syst Rev (4): CD003375.

[3] Saunders, J., Hungerford, B., Wisbey-Roth, T., Lucas, P., and Wilson, S. 2010. "The Use of Prolotherapy in the Sacro-Iliac Joint.” Br. J. Sports Med 44 (2): 100-4.

[4] Soliman, D. 2016. Pain Management by Prolotherapy and Perineural Injection therapy. Germany: Lambert Academic Publishing, 13-38.

[5] Rabago, D., Jeffery, J., Marlon, M., Richard, K., Jessica, G., Neil, A., and Aleksandra, Z. 2013. "Dextrose Prolotherapy for Knee Osteoarthritis: A Randomized Controlled Trial.” Annals of Family Medicine 11 (3): 229-37.

[6] Hackett, G., Hemwall, G., and Montgomery, G. 1956. Ligament and Tendon Relaxation Treated by Prolotherapy. Springfield Illinois U.S.A.: Charles C Thomas, 3-6.

[7] Lyftogt, J. 2007. "Subcutaneous Prolotherapy Treatment of Refractory Knee, Shoulder and Lateral Elbow Pain.” Aust Musculoskeletal Med 12 (2): 110-2.

[8] Banks, A. 1991. “A Rationale for Prolotherapy.” Journal of Orthopedic Medicine 13 (3): 1-10.

[9] Lyftogt, J. 2010. “Neural Prolotherapy.” Presented at the Hackett Hemwall Foundation and the Italian Society for Prolotherapy, Ferrara, Italy.

[10] Adam, D. 2011. “Neural Prolotherapy.” Journal of Prolotherapy 3 (2): 639-43.

[11] Zambrano, J., Chirinos, J., Chakko, S., et al. 2010. "Migraine Pain Results from Neurogenic Inflammation Affecting Cranial Blood Vessels and Dura.” Neurology 75 (11): 960-6.

[12] https://en.wikipedia.org/wiki/Vitamin_D.

[13] Minghetti, P., and Norman, A. 1988. “1,25(OH)2-Vitamin D3 Receptors: Gene Regulation and Genetic Circuitry.” FASEB J. 2 (15): 3043-53.

[14] Ameri, P., Giusti, A., Boschetti, M., Murialdo, G., Minuto, F., and Ferone, D. 2013. "Interactions Between Vitamin D and IGF-1: From Physiology to Clinical Practice.” Clin Endocrinol 79 (4): 457-63.

[15] Bellamy, N., Bell, M., Goldsmith, C., Pericak, D., Walker, V., Raynauld, J., Torrance, G., Tugwell, P., and Polisson, R. 2005. "Evaluation of WOMAC 20, 50, 70 Response Criteria in Patients Treated with Hylan G-F 20 for Knee Osteoarthritis.” Ann Rheum Dis 64 (6): 881-5. 